\title{
Arnaud Genon, Autofiction: pratiques et théories. Articles
}

\section{Francesca Forcolin}

\section{(2) OpenEdition}

1 Journals

\section{Edizione digitale}

URL: http://journals.openedition.org/studifrancesi/2333

DOI: 10.4000/studifrancesi.2333

ISSN: 2421-5856

\section{Editore}

Rosenberg \& Sellier

\section{Edizione cartacea}

Data di pubblicazione: 1 aprile 2014

Paginazione: 204-205

ISSN: 0039-2944

\section{Notizia bibliografica digitale}

Francesca Forcolin, « Arnaud Genon, Autofiction: pratiques et théories. Articles », Studi Francesi [Online], 172 (LVIII | I) | 2014, online dal 01 avril 2014, consultato il 18 septembre 2020. URL : http:// journals.openedition.org/studifrancesi/2333; DOI : https://doi.org/10.4000/studifrancesi.2333

Questo documento è stato generato automaticamente il 18 settembre 2020.

\section{(c) (i) $\odot$}

Studi Francesi è distribuita con Licenza Creative Commons Attribuzione - Non commerciale - Non opere derivate 4.0 Internazionale. 


\title{
Arnaud Genon, Autofiction: pratiques et théories. Articles
}

\author{
Francesca Forcolin
}

\section{NOTIZIA}

ARNAUD GENON, Autofiction: pratiques et théories. Articles, Paris, Mon Pétit Editeur, 2013, p.

224.

1 È dagli anni settanta che si parla di autofiction, precisamente dal 1977, quando il neologismo comparve per la prima volta sulla quarta di copertina di Fils, firmato Serge Doubrovsky. Da allora questo genere letterario, inteso come una delle forme contemporanee dell'autobiografia, ha nutrito il dibattito critico, dando vita a due schieramenti contrapposti: i pro e i contro, i difensori e gli avvocati del diavolo.

2 È da più di dieci anni che Arnaud GENON si occupa di autofiction, e in questo lasso di tempo ha pubblicato - in riviste on-line, cartacee, universitarie e non - una cinquantina di articoli che illustrano le numerose pubblicazioni sul tema. Oggi l'A. decide di riunire questi articoli - ai suoi occhi una vera e propria "cartographie de l'autofiction" - con l'obiettivo di offrire al lettore una panoramica, una visione globale di questo genere ambiguo che mescola vita e scrittura, realtà e finzione, proponendo una sintesi delle diverse accezioni del termine e mostrando il dinamismo di un "concetto" che, facendo tanto parlare di sé nel bene o nel male, fa parlare di letteratura. L'A. rifiuta la volontà di darne un'ennesima definizione, anche se, nelle pagine introduttive (pp. 7-11), all' autofiction viene dato lo statuto di uno "strumento" che il soggetto utilizza per interrogare se stesso, «refuser l'idée d'une vérité univoque et revendiquer sa fracture». Il volume è diviso in due parti: la prima (Pratiques) raggruppa quegli articoli che propongono un'analisi delle ultime opere (o romanzi, poiché l'appartenenza al genere della finzione, così come l'uguaglianza identitaria tra autore, narratore e personaggio, è un requisito necessario perché un testo sia autofictif, ed è qui che risiede tutta la sua contraddizione) dei maggiori esponenti autofictionnistes, o etichettati tali dalla critica. 
Troviamo 32 articoli o "appunti", molto brevi (dalle due alle sei pagine), classificati per autore e per ordine alfabetico, che permettono al lettore di farsi un'idea di alcune produzioni contemporanee, lasciandogli tuttavia un ampio margine di critica - non tanto recensioni ma spunti, idee, bagliori, che ponendo interrogativi stimolano la riflessione. Apre la sezione l'analisi delle ultime pubblicazioni di Christine Angot: il primo articolo è Christine Angot: l'écriture ou la vie (pp. 15-21), segue Angot au rendez-vous (pp. 23-24), Les frontières de l'amour (pp. 25-27), Le style avant tout (pp. 29-30). Genon passa poi al secondo autore, Frédéric Beigbeder, con l'articolo Retour en enfance (pp. 31-32); a Samuel Benchetrit, con Chroniques d'un branleur" (pp. 33-34) e Les amours de la bande à Bench (pp. 35-36); Fanny Cosi, con Penser les plaies (pp. 37-38); Alain Defossé, con Du fait divers comme écriture de soi (pp. 39-40); Christophe Donner con Le roi Donner (pp. 41-42) e Hommage au beau-père (pp. 43-44); Serge Doubrovsky con Son testament littéraire (pp. 45-47); Vincent Eggericx con De l'art d'écrire à l'arc (pp. 49-50); Frederick Exley con Alcool, femmes et foot (pp. 51-53); Claire Fercak con De l'autofiction comme quête (pp. 55-56); Georges-Arthur Goldschmidt con Les aventures singulières de G-A Goldschmidt (pp. 57-58) e Vivre après (pp. 59-60); Isabelle Grell con l'articolo dal titolo L'autofiction dans le boudoir (pp. 61-62); Laurent Herrou con Cocktail Molotov (p. 63); Alexandre Jardin con L'Historie comme miroir (pp. 65-67); Alexandre Lacroix con Humain, trop humain? (pp. 69-70); Dany Laferrière con Haiti cœur du monde (pp. 71-72); Camille Laurens con «Le roman vrai» de Camille Laurens (pp. 73-74); Claire Legendre e Jérôme Bonnetto con Roman (d'amour) photo (pp. 75-77); Daniel Mendelsohn con Une archéo-sociologie du moi (pp. 79-80); Philippe Mezescaze con Mots et maux de l'amant (pp. 81-82); Marie Nimier con Décousu et beau comme la vie (pp. 83-84); Ann Scott con Ann Scott à l'heure du bilan (pp. 85-86); Antoine Silber con Le livre de la mère (pp. 87-88); Mathieu Simonet con Renaître de ses cendres... (pp. 89-91); infine, i due articoli sulla scrittura di Abdellah Taïa, Le baraka d'Abdellah Taïa (pp. 93-94) e Une écriture de soie (pp. 95-97).

4 La seconda parte del volume (Théories) scava all'interno del dibattito critico generato dall'autofiction: l'A. riprende alcune pubblicazioni in cui il «mauvais genre» viene analizzato in maniera teorica - quei testi fondamentali, bibliografia di base per la sua comprensione e approfondimento. Qui, i testi studiati non sono presentati secondo l'ordine scelto per la prima parte: Genon tratta inizialmente le opere più generali e teoriche. E tra queste troviamo uno studio di Philippe Gasparini, analizzato nell'articolo che porta il titolo Une mise au point salutaire (pp. 101-105); di Vincent Colonna, nell'articolo Autofictions (pp. 107-110); Arnaud Schmitt, De l'autofiction à l'autonarration (pp. 111-116); Jean-Louis Jeannelle, Catherine Viollet e Isabelle Grell, in Les coulisses de l'autofiction (pp. 117-123); Philippe Vilain, in Voyage en autobiographies (pp. 125-126); Philippe Vilain, in Autofiction: la théorie du romancier (pp. 127-129); Chloé Delaume, in De l'autofiction expérimentale (pp. 131-133); Philippe Forest, in Moi, je... (pp. 135-138); Joël Zufferey, in Variations autofictionnelles (pp. 139-144); Roger-Michel Allemand e Christian Milat, in Pour une nouvelle autobiographie (pp. 145-149); Pierre Brunel, in L'inépuisable moi (pp. 151-155); Kamel Riahi, in Le 'pacte autobiographique' s'exporte (pp. 157-159); AnneRachel Hermetet e Jean-Marie Paul, in Le 'moi' dans tous ses états (pp. 161-169); Madeleine Ouellette-Michalska, in L'autofiction sans frontières (pp. 171-174).

5 L'interesse di questi brevi ma densi articoli risiede in una panoramica del testo scelto, generica e di facile comprensione, e in un paragrafo finale riassuntivo che permette, con un colpo d'occhio, di capire come l'autofiction viene concepita dallo scrittore in questione. Gli ultimi articoli dell'A. propongono la lettura di testi che analizzano autori specifici e la relazione che questi intrattengono con l'autofiction, da Proust a Colette a 
Beigbeder. Troviamo l'articolo dal titolo De l'autofiction comme outil herméneutique (pp. 175-181) che verte su uno studio di Thomas Carrier-Lafleur; De la photographie en autofiction (pp. 183-186) su Roger-Yves Roche; Antoine d'Agata: de la photographie 'comme art funambole' (pp. 187-189) su Antoine d'Agata e Christine Delory-Momberger; Serge Doubrovsky, témoin de son époque (pp. 191-194) su Patrick Saveau; Du côté de chez Doubrovsky (pp. 195-200) su Elizabeth H. Jones; Camille Laurens: radiographie d'une œuvre (pp. 201-204) sulle teorie di Florent Georgesco e Camille Laurens; L'œuvre de Colette: de l'autobiographie à l'autofictionnel (pp. 205-208) su Stéphanie Michineau; Frédéric Beigbeder (pp. 209-212) su Alain-Philippe Durand. Chiude L'autofiction dans le monde arabe, conversazione tra Genon e M'hamed Dahi (pp. 213-221). 